\title{
CORSI E CONGRESSI
}

Aprile 2008

XI CONGRESSO NAZIONALE CROI

2-5 Aprile 2008 - Bologna

Segreteria Organizzativa: Dynamicom

via San Gregorio 12, Milano

Tel. 02.89693766 - Fax 02.201176

E-mail: mortara@dynamicommunications.it

Sito web: www.croi.it

THE FIFTH INTERNATIONAL CONGRESS ON FAMILIAL MEDITERRANEAN FEVER AND SYSTEMIC AUTOINFLAMMATORY DISEASES 4-8 Aprile 2008 - Roma

Segreteria scientifica: M. Gattorno,

C. Cerquaglia, V. Curigliano

E-mail: fmfsaid08@pyramide.it

Segreteria organizzativa: Pyramide S.r.l.

Via San Godenzo 164, 00189 Roma

Tel. 06.3314114 - Fax 06.3314113

E-mail: segr-fmfsaid08@pyramide.it

Sito web: www.fmfsaid2008.com

VII CORSO TEORICO PRATICO:

L'ANALISI DEL LIQUIDO SINOVIALE

10-11 Aprile 2008

Abbazia di Praglia, Padova

Segreteria scientifica: Francesca Oliviero,

Roberta Ramonda

Cattedra e UOC di Reumatologia,

Università di Padova

Tel. 049.8212199 - Fax 049.8212191

Segreteria organizzativa: Nadirex Internaltional Srl

Via Riviera 39, Pavia

Tel: 0382.525714/35 Fax: 0382.525736

E-mail: info@nadirex.com

\section{CORSO TEORICO-PRATICO LA} SETTIMANA DELLE MALATTIE RARE IN REUMATOLOGIA "ALESSANDRO LUPOLI" 16-22 Aprile 2008 - Pisa

Segreteria organizzativa:

Clinical and Experimental Rheumatology

Via Santa Maria 31, 56126 Pisa

Tel. 050-40124 - Fax 050-502299

E-mail: marconcini@ clinexprheumatol.org

Sito web: http://www.clinexprheumatol.org/rare3
Maggio 2008

7TH EUROPEAN LUPUS MEETING

7-10 Maggio 2008 - Amsterdam

Informazioni: www.lupus2008.it

HENRY KUNKEL SOCIETY ANNUAL MEETING 2008:

Autoimmunity, inflammation and lymphoproliferative diseases

21-24 Maggio 2008 - Santa Margherita Ligure Segreteria organizzativa: Accademia Nazionale di Medicina

Via Martin Piaggio 17/16 - 16122 Genova

Tel. +39010 83794225 - Fax +3901083794260

E-mail: registration@accmed.org

Sito web: www.henrykunkelsociety.org

Giugno 2008

15TH EULAR SONOGRAPHY CORSE

8-11 Giugno 2008 - Parigi

Informazioni: www.eular.org

Settembre 2008

6TH INTERNATIONAL CONGRESS ON

AUTOIMMUNITY

3-7 Settembre 2008 - Porto, Portogallo

Segreteria organizzativa: Kenes International, $1-3$ rue de Chantepoulet, Svizzera

Tel. +41229080488 - Fax +4122732 2850

E-mail: autoimmunity@kenes.com

Sito web: www.kenes.com/autoimmunity

3RD EULAR COURSE: CAPILLAROSCOPY AND RHEUMATIC DISEASES

15-17 Settembre 2008 - Genova

Segreteria scientifica: Maurizio Cutolo

Cattedra di Reumatologia, Università di Genova

Tel. 010.3537994 - Fax 010.3538885

E-mail:mcutolo@unige.it

Segreteria organizzativa:

Edra Spa, viale Monza 133, Milano

Tel. 02.28172 300 - Fax 02.28172 399

E-mail: 3rdcapillaroscopy@edraspa.it

Sito web: www.eular.org

\section{OARSI WORLD CONGRESS}

18-21 Settembre 2008

Roma, Marriott Park Hotel

Informazioni: www.oarsi.org 


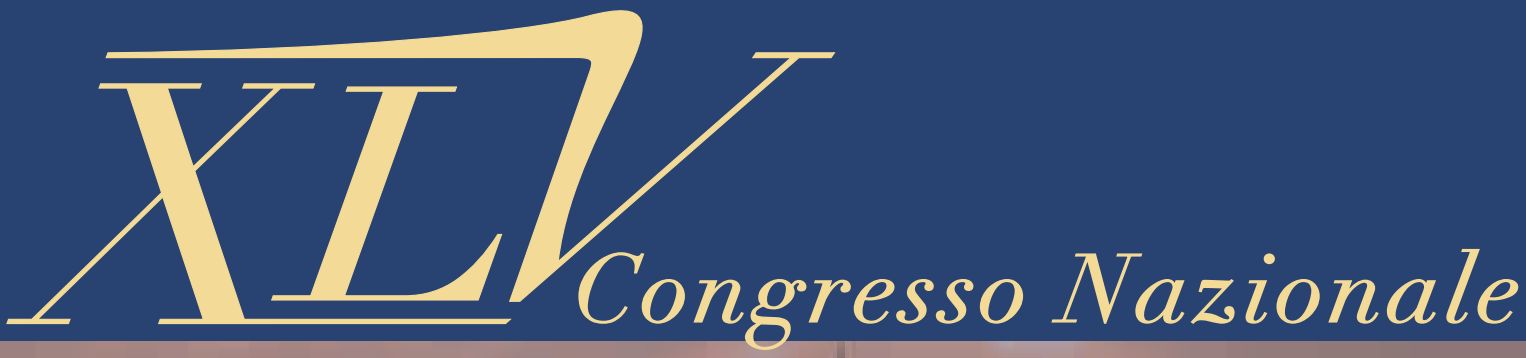

\section{della Società Italiana di Reumatologia}

\section{Venezia 15118 Ottobre \\ Palazzo del Cinema}

Deadline abstract 30 maggio 2008

Deadline quota di registrazione ridotta 20 giugno 2008

Il programma del XLV Congresso Nazionale della Società Italiana di Reumatologia è presente in Internet: www.reumatologia.it 


\section{PREMI DI RICERCA SUI TEMI}

La terapia di combinazione leflunomide e biologici nel paziente affetto da artrite reumatoide.

COMMISSIONE SCIENTIFICA

Prof. M. Cutolo (Genova)

Prof. S. Bombardieri (Pisa)

Prof. C. Montecucco (Pavia)

Dott. M. Confalonieri (sanofi-aventis)
La safety della leflunomide in monoterapia e in associazione con biologici e la sua gestione nella pratica clinica quotidiana.

Le differenze tra paziente maschio e femmina nella risposta alle diverse terapie con DMARDs singoli o in associazione.

APERTO A TUTTI GLI SPECIALISTI IN REUMATOLOGIA CHE LAVORANO SUL TERRITORIO NAZIONALE

La terapia dell'artrite reumatoide è profondamente cambiata negli ultimi anni grazie all'avvento di nuovi principi attivi e di nuovi approcci di trattamento che prevedono l'uso concomitante di più farmaci.

II razionale della terapia di combinazione si basa sul concetto che farmaci con meccanismi d'azione complementari possano produrre un effetto terapeutico sinergico, con una diminuzione dei dosaggi dei singoli principi attivi e conseguente riduzione del rischio di effetti collaterali.

La terapia di combinazione leflunomide e biologici risulta ad oggi poco studiata: mancano informazioni relative al livello di efficacia, alla tipologia di pazienti che potrebbero beneficiare di questo approccio terapeutico e al profilo di sicurezza.

Al fine di aumentare le conoscenze su questi temi clinici di stretta attualità, nell'ambito delle sue attività di supporto alla ricerca

\section{sanofi-aventis indice un bando di concorso per}

I premi sono destinati alla realizzazione di lavori scientifici originali, retrospettivi o prospettici.

Per poter concorrere all'assegnazione dei premi è necessario far pervenire esplicita richiesta di partecipazione, firmata da tutti gli autori del lavoro, alla Segreteria Scientifica via e-mail o tramite posta raccomandata R/R entro e non oltre il 30 giugno 2008 unitamente ai seguenti documenti:

- Lavoro originale in lingua italiana che non dovrà superare le venti cartelle dattiloscritte (carattere: times new roman 12) comprensive di tabelle, grafici, bibliografia e indicazione esplicita del primo autore.

- Consenso scritto al trattamento dei dati personali ai sensi del D.Lgs. 196/03 (Codice in materia di protezione dei dati personali).

Tutti i lavori originali pervenuti entro la data stabilita verranno giudicati da una Commissione Scientifica, costituita da: Prof. M. Cutolo (Università di Genova); Prof. S. Bombardieri (Università di Pisa-Past president SIR); Prof. C. Montecucco (Università di Pavia-President SIR); Dott. M. Confalonieri (Responsabile area Osteoreuma direzione medica sanofi-aventis).

II giudizio di merito fornito dalla Commissione sarà insindacabile.

I lavori scientifici saranno valutati in base ai seguenti criteri:

- Originalità dei risultati

- Completezza dell'analisi

- Coerenza tra risultati e conclusioni

- Utilità nella pratica clinica delle conclusioni

La cerimonia di premiazione dei vincitori avverrà al termine dei lavori dell'evento "AREA 2008", previsto per il 20-21 settembre 2008.

La Segreteria Scientifica comunichèrà ai vincitori il luogo in cui si svolgerà l'evento "AREA 2008" unitamente alle istruzioni per il ritiro del premio.

II presente bando scade il 30 giugno 2008

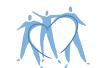

\section{sanofi aventis}

La salute, la cosa più importante

\section{Segreteria Scientifica}

"Bando Reumatologia Sanofi-Aventis" AIM Group - AIM Education s.r.I. Sede di Milano

Via Ripamonti, 129 - 20141 Milano

Tel. 02566011 - Fax 0256609049

E-mail:

reumatologia.bando.sa@aimgroup.it sanofi-aventis S.p.A.

Viale Luigi Bodio 37/b 20158 Milano 